\title{
浅谈 ADS-B 信号对 A-SMGCS 系统的影响
}

\section{The Influence of ADS-B Signal on A-SMGCS System}

\section{王雪}

Xue Wang

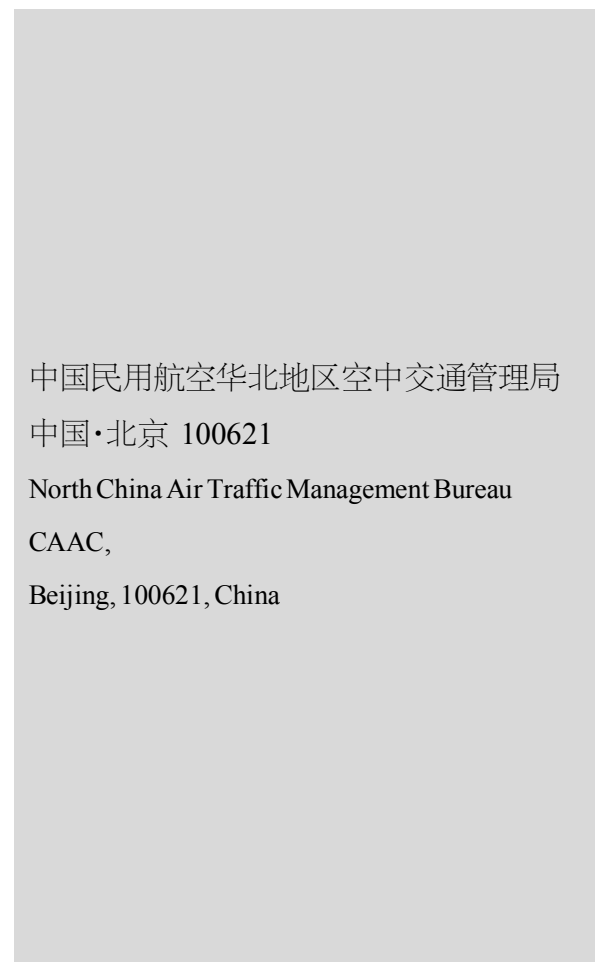

【摘要】随着中国航空运输业的快速发展,机场规模逐步扩大, 日趋复杂的机场场面活动 已成为影响机场飞行安全和运行效率的重要因素。A-SMGCS 系统作为辅助塔台管制进行 机场场面活动管理和指挥的重要系统工具,对当前的空中交通管理具有重要的意义,同时 广播式自动相关监视 (ADS-B)信号作为 A-SMGCS 系统监视源的重要数据来源之一, 它的 分裂和偏移将对管制指挥造成非常大的影响。论文将从原理上分析 ADS-B 信号分裂产生 的原因, 以及对 A-SMGCS 系统的影响。

【Abstract】With the rapid development of China's air transportation industry, the scale of airports has gradually expanded, and increasingly complex airport surface activities have become an important factor affecting airport flight safety and operational efficiency. The A-SMGCS system is an important system tool for assisting tower control in airport surface activity management and command. It is of great significance to the current air traffic management, and broadcast-type automatic related monitoring (ADS-B) signal is one of the important data sources of the A-SMGCS system monitoring source, and its split and deviation will have a great impact on the control command. This article will analyze in principle the reasons for the ADS-B signal split and the impact on the A-SMGCS system.

【关键词】A-SMGCS 系统;ADS-B 信号;GPS 信号;管制

【Keywords】A-SMGCS system; ADS-B signal; GPS signal; regulation

【DOI】10.36012/etr.v2i4.1661

\section{1 引言}

随着中国航空运输业的迅猛发展, 大型枢纽机场日均飞 机起降架次快速增长, 交通密度较大且保持持续升高的态 势 , 机场运行环境更加复杂 , 在机场运行中处理好飞机与飞 机、飞机与车辆之间的冲突, 避免出现地面交通混乱所导致 的机场运行安全水平和运行效率下降问题变得非常重要。ASMGCS 系统作为集场面监视、地面管制、冲突告警、路由规 划、灯光引导等功能于一体, 并利用电子进程单实现多塔台 协调合作的机场场面引导系统优势明显, 被广泛应用于大型 枢纽机场。
ADS-B 系统是基于 GPS 卫星定位和地/空数据链通信的 航空器监视系统, 由于其具有定位精准度高、数据更新率快, 监视能力强等特点,被应用于 A-SMGCS 系统中。ADS-B 系统 输出的目标监视数据是 A-SMGCS 系统实现场面监视功能, 对在机场场面的航空器和车辆进行连续定位和标识的重要数 据源之一, 如果 ADS-B 信号出现分裂或者偏移现象, 则会造 成 A-SMGCS 系统态势界面显示屏上出现信号分裂和偏移现 象, 由此导致 DUPE 告警、RIW 告警、TCW 告警等多个告警产 生, 同时造成部分航班灯光引导、路由规划功能失效。因此, 解 决 ADS-B 信号偏移和分裂问题对提高管制工作效率和保障 机场场面航空器的安全性具有非常重要的作用。 


\section{A-SMGCS 系统总体介绍}

\section{1 系统概述}

A-SMGCS 系统是一套实时处理系统，每天 24 小时不间 断运行, 为管制员提供地面管制的自动化手段。设计中采用国 际上广泛使用并得到公认的设计思想和设计原理，进行模块 化、多重呪余的优化设计。系统设计采用分布式、客户/服务器 (C/S )、开放式结构。系统采用局域网作为系统内部信息传输、 交互的基本通道,其中的双局域网为互为备份的主工作网,第 三网用作系统维护、数据发布和重演。系统采用了各种可靠性 措施, 特别是双机主/从热备份的体系结构, 具有很高的可用 性指标。

\section{2 系统的主要功能}

A-SMGCS 系统能够连续的跟踪、识别航空器和车辆等运 动目标, 为地面管制提供实时的场面动态。其主要接收场面监 视雷达 (SMR)、多点相关监视 (MDS) 和广播式自动相关监视 (ADS-B) 等监视设备输出的目标监视数据, 通过多元数据融 合处理, 对在机场场面覆盖范围内运动的目标进行连续的定 位和标识，在管制员的监视界面上显示机场场面运动目标的 运行态势和运行环境。因为机场运行网络的复杂性和运行的 动态性导致了场面运动中的不确定性, 所以 A-SMGCS 系统 需要实时进行各种运行告警的计算, 并且提供了直观的画面 或声音告警提示以辅助管制员进行管制,包括冲突告警、目标 丢失告警、重复二次代码告警等。A-SMGCS 系统还具备滑行 路线自动选择能力, 能够为相关的航空器和车辆提供最佳路 由,对其场面滑行路线进行自动安排。A-SMGCS 系统除了上 述地面系统必备的功能外, 还具备助航灯光引导数据的生成 能力, 根据系统规划的场面滑行路径、目标的实时运动位置和 跑道视程等数据 ${ }^{[1]}$

\section{ADS-B 技术概况}

\subsection{ADS-B 技术简介}

广播式自动相关监视系统 (ADS-B) 是一种基于卫星定位 和利用空一地、空一空数据链通信完成交通监视和信息传递 的空管监视新技术; 是航空器通过广播模式数据链, 自动提供 由机载导航设备和卫星定位系统生成的数据（包括航空器识 别、定位及其他相关的附加数据), 地面设备、其他航空器及 管制等相关部门通过航空数据链接收飞机的广播信息,形成 了由卫星系统、飞行器及地基系统组成的高速数据链空地一 体化协同监视体系, 供飞行人员或管制人员监视航空器运行 状态 ${ }^{[2]}$ 。

\subsection{ADS-B 系统组成和技术功能特点}

ADS-B 系统包含机载和地面设备两部分, 机载设备由 GPS 接收机及 UAT 收发信机(GDL-90)、一根 GPS 接收天线、 两根 UAT 天线、气压高度编码器、驾驶舱显示器等组成。机载 设备依靠 GPS 来确定飞机的精确位置, 然后将位置信息转换 成数字码, 该数字码结合了其他一些信息(如速度、高度及飞 机是否转弯、爬升或下降), 每秒更新数次, 在适当的频率通过 数据链从飞机广播出来, 在 $100 \mathrm{n}$ mile 内的其他飞机就能自动 识别互相的位置, 可自我保持间隔。

地面基站主要包括地面收发机(GBT)及其天线、ADS-B 数据服务器、控制显示计算机(客户端)、配套的网络设备、主 服务器等。其主要功能是 GBT 与数据链接收天线相连用于接 收机载 ADS-B 系统发出的广播信号, 数据服务器对接收到的 ADS-B 信号进行解码和数据处理, 转换为计算机网络上的 UDP 协议数据包, 提供可供客户端使用的信号。地面 ATC 对 终端和航路飞行的飞机实施监控指挥; 机场场面活动的飞机、 车辆间保持安全间隔, 起到场监监视作用, 且能有效地用在偏 远地区或山区, 这些以往雷达无法覆盖或受限制的地区。

\section{4 造成 ADS-B 信号不稳定的原因分析}

(1)GPS 系统是 ADS-B 系统中飞行器定位数据的唯一来 源, 因此在系统运行过程中, 若出现 GPS 卫星信号受到干扰, 或 GPS 卫星出现故障, 将导致 ADS-B 系统无法正常工作。 GPS 作为 GNSS（国际民航组织标准化的全球卫星导航系统） 的重要组成部分, 其导航定位及授时功能广泛应用于航空系 统。目前，民航空管系统及行业各相关用户普遍采用了以 GNSS 信号精确授时为基础的时间同步策略和措施方法, 以 满足运行信息交互操作中的精确时间标记和运行协调计时等 需求。其中, 以 GPS 信号作同步时钟、定位信息的用户占有很 大的比重 ${ }^{[3]}$ 。

(2)民用航空 GPS 目前使用的三个频段分别是: L1 波段, 频率为 $1575.42 \mathrm{MHz}$; L2 波段, 频率为 $1227.6 \mathrm{MHz}$; L5 波段, 频

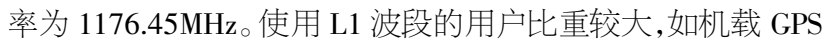
导航设备、空管地面 GPS 授时设备。

(3)机载 GPS 接收系统对自身定位的精度完全受限于卫 星群位置和接收的 GPS 信号质量。目前中国使用的 ADS-B OUT 技术体制的地面接收设备只能被动使用下行的位置数 据, 即接收机载设备发送的位置信息, ADS-B 地面设备无法 进一步提高飞机的位置精度。当机载设备接收的 GPS 信号受 干扰时, 会导致自身定位精度严重下降, 并将下降的位置精度

(下转第 37 页) 
主要是对设计成果、施工数据、施工资源及进度进行展示; 档 案交付包括对竣工资料的管理、签署和生成等。因此,在业务 服务层, 可以利用企业 IT 基础设施和微服务生态体系逐步形 成的基础服务, 梳理并优化管道建设期业务, 合理设计并划分 业务边界, 对基础服务进行编排、组和,逐步形成支持不同业 务领域的微服务业务组件。

\section{5 综合应用层}

综合应用层主要包括：对管道项目的进度监控、视频监 控、焊接工况监控、质量安全管理和应急指挥。在综合应用 层, 充分利用微服务组件及微服务治理框架, 结合数据共享中 心提供的数据资源及开放的数据接口, 深度分析挖掘各业务 职能部门及业务相关方需求, 形成功能丰富、数据全面、展示 方式多样化、可扩展性及实用性强的智能化平台, 为管道建设 期信息化管理提供数据支撑和辅助决策。

\section{6 应用终端}

该层的作用是通过使用工作站、各种移动端、桌面 PC 端 及专用设备等进行业务应用布局, 通过系统提供的管道建设 期业务微服务组件及智能化基础平台, 根据业务需求, 进行服 务编排、组装、调度, 形成对工作站、平板、大屏及移动应用等
多种终端的支持, 满足用户对不同操作终端办公的需要, 满足 智慧管道智能系统的应用。

\section{4 结语}

目前, 随着数字管道建设的迅速发展, 智慧管道将成为未 来中国管道发展的重点方向, 尤其是天然气智慧管道的建设 将成为重点。因此, 应全面贯彻“全数字化移交, 全智能化运 行, 全生命周期管理” 的智慧管道建设理念, 最终实现智慧管 道在运行中具有 “信息感知、自动预判、智能优化、自我调节” 的功能。

\section{参考文献}

[1]程万洲,王巨洪,王学力, 等. 我国智慧管道建设现状及关键技术 探讨[J].石油科技论坛,2018(3):34-39.

[2]王昆,李琳,李维校.基于物联网技术的智慧长输管道[J].油气 储运,2018,37(1):15-19.

[3] 李海润.智慧管道技术现状及发展趋势[J].天然气与石油, 2018,36 (2):129-132.

[4]李秋扬,赵明华,任学军,等.中国油气管道建设现状及发展趋势 [J].油气田地面工程 2019,38(Z1):14-17.

\section{(上接第 34 页)}

通过数据链广播至 ADS-B 地面接收设备, 导致 ADS-B 系统 信号质量出现偏移。

(4)由于运输航空飞行器 GNSS 天线通常安装在顶部, 由 于飞行器金属机体的屏蔽作用, 高空飞行时, 干扰源与飞行器 GNSS 天线之间不易形成通视，干扰信号较难进入 GNSS 天 线, 出现干扰的状况较少。飞行器机动时, GNSS 天线接收到 的信号会变弱, 由于翻滚角的存在, 也更容易与可能存在的干 扰源形成通视, 出现被干扰的状况。飞行器在五边的时候, 由 于高度连续降低, 也容易受到地面 GNSS 干扰信号的影响。

\section{5 减少对 A-SMGCS 系统信号影响的建 议及措施}

多次出现民航 GPS 信号受到干扰的现象, 对空管设备运 行造成了潜在的风险, 各空管运行单位针对 GPS 信号干扰的 问题应引起重视, 并制定相应的应急措施, 必要时可以在 ASMGCS 系统中断开受干扰影响的 ADS-B 监视信号源, 确保 A-SMGCS 系统的综合航迹不受其影响, 并将干扰情况及时通 报当地无线电管理委员会协助其查明干扰源, 采取措施消除 干扰。对 A-SMGCS 系统的信号源进行区域性调整, 对于某些
特定位置 ADS-B 数据不稳定的区域, 系统不再使用其信号数 据, 加入新的防分裂算法, 并且设定信号抑制区域, 以保证 ASMGCS 系统综合航迹信号稳定。

\section{6 结语}

综上所述, ADS-B 在应用于 A-SMGCS 系统方面具有诸 多技术优势, 为场面监控系统提供了一种更加有效的选择。但 在实际运行中, 因为 GPS 信号干扰问题, ADS-B 信号出现偏 移和分裂, 故通过技术手段排余 GPS 信号干扰源尤为重要。 解决 ADS-B 信号偏移和分裂问题, 使 A-SMGCS 系统的目标 监视数据融合后稳定输出, 将有效确保机场地面制辖区内的 飞行安全,提高飞行效率, 满足机场的管制需求,对提升机场 运行安全和效益具有重要作用。

\section{参考文献}

[1]曾思弘.A-SMGCS 系统设计分析与实现[J]. 智能建筑与城市 信息,2015(9):73-75+78.

[2]罗文田.ADS-B 机场场面监视技术研究[J].中国民航飞行学院 学报,2011(2):67-90.

[3]胡飞.ADS-B 系统的原理分析与风险研究 [J]. 科技传播, 2011 (3):94. 